\title{
Interfacial Water Properties in the Presence of Surfactants
}

\author{
Naga Rajesh Tummala ${ }^{1 \#}$, Shi Liu ${ }^{2}$, Dimitrios Argyris $^{3}$, and Alberto Striolo ${ }^{4}$ \\ ${ }^{1}$ School of Chemistry and Biochemistry, \\ Georgia Institute of Technology, \\ Atlanta, GA \\ ${ }^{2}$ Halliburton Inc, \\ Houston, TX \\ ${ }^{3}$ Applied Materials Inc, \\ Santa Clara, CA \\ ${ }^{4}$ Department of Chemical Engineering \\ University College London, London, UK
}

Key words: Interfacial water, molecular dynamics, surfactants, electrostatics

${ }^{\#}$ Author to whom all correspondence should be addressed

Email: nagarajesh@gatech.edu 


\begin{abstract}
Water, due to its fundamental role in biology, geology, and many industrial applications, and due to its anomalous behavior compared to that of simple fluids, continues to fascinate and attract extensive scientific interest. Building on previous studies of water at contact with different surfaces, in this study, we report results obtained from molecular dynamics simulations of water near hydrophilic and hydrophobic interfaces in the presence of non-ionic and ionic amphiphilic molecules, hexaethylene glycol monododecyl ether $\left(\mathrm{C}_{12} \mathrm{E}_{6}\right)$ and sodium dodecyl sulfate (SDS). We elucidate how these surfactants affect packing (i.e., density profiles) and orientation of interfacial water. The results highlight the interplay of both surfactant charges and substrate charge distribution predominantly on the orientation of water molecules, up to distances larger than those expected based on simulation results on flat solid surfaces. We also quantify the dynamics of interfacial water molecules by computing the residence probability for water at contact with the various substrates. We compare our results to those previously obtained for interfacial water on silica and graphite and also with experimental sum-frequency vibrational spectroscopy results at air-water interface in the presence of surfactants. Our analysis could be useful for better understanding interfacial water not only near solid substrates, but also near selfassembled/aggregated molecules at a variety of interfaces.
\end{abstract}




\section{Introduction}

Water molecules next to different surfactant aggregates in the bulk have been studied experimentally $^{1-4}$ and through simulations. ${ }^{5-6}$ The effect of self-assembled monolayers with different head groups on interfacial water was also studied extensively. ${ }^{7-8}$ On the contrary, the molecular level properties of water molecules next to surfactants adsorbed at different solidliquid interfaces did not receive much attention, although recent results from the study of specific ion effects ${ }^{9-10}$ indicate that surfactants with different head groups can alter the properties of interfacial water to different extents. Understanding water solvation of various self-assembled aggregates is necessary for predicting and perhaps manipulating a number of macroscopic substrate properties. ${ }^{11}$ Among experiments used to assess the properties of interfaces, vibrational sum frequency generation (VSFG) is widely used to study the orientation of water at interfaces, ${ }^{12-14}$ while adsorption ${ }^{15-16}$ and scattering experiments ${ }^{17-20}$ are performed to determine the structure of adsorbed surfactant layers. Surfactants can aggregate on a surface, causing a decrease in the density of interfacial water because of excluded-volume effects. The present article stems from the hypothesis that changes in local density do not necessarily imply changes in the properties of interfacial water molecules. Related to this hypothesis, it is also possible that the formation of disordered self-assembled surfactant structures yield interfacial water properties that differ compared to those of interfacial water without surfactants.

The relaxation time characteristic for surfactant self-assembly and water rearrangement are different (the former much longer than the latter), conducting sufficiently long molecular dynamics simulations $^{5,2122-23}$ allows us to quantify both phenomena. The results obtained for surfactants on silicon oxide and graphite surfaces were reported previously. ${ }^{24}$ Herein we discuss how water can be simultaneously affected by both surface and surfactants. 
Our results are quantified in terms of water density profiles near the various surfaces, dipole moment distribution of interfacial water molecules, and hydration structure of the head groups of both ionic and nonionic surfactants close to, and away from the interface. Since the dynamics of interfacial water molecules determine a variety of processes related to corrosion, the ability of small molecules to diffuse to and near interfaces, the performance of coatings, and many more, we also report the residence probability and the reorientational dynamics of water molecules at the various interfaces considered.

\section{Simulation Methodology}

The trajectories from the simulations reported in our recent article ${ }^{24}$ are utilized to analyze the behavior of water next to surfactants sodium dodecyl sulfate (SDS) and hexaethylene glycol monododecyl ether $\left(\mathrm{C}_{12} \mathrm{E}_{6}\right)$ adsorbed at silica and graphite surfaces. Simulations of SDS at the graphite-water interface were performed at multiple SDS surface densities. These simulations are referred to as X_GRA, where $X$ corresponds to the surface area, in $\AA^{2}$, available for each surfactant (large surface areas correspond to low surface densities).

In addition to graphite, we simulated model surfaces obtained from silicon dioxide. These surfaces differ in the density of $\mathrm{OH}$ groups exposed to water. We considered a silica surface with 13.6 non-bridging $\mathrm{O}$ atoms per $\mathrm{nm}^{2}$, which we refer to as high-density silica (HD), and a silica surface with 4.5 non-bridging $\mathrm{O}$ atoms per $\mathrm{nm}^{2}$, low-density silica (LD). For both the HD and LD silica surfaces we considered $100 \%, 50 \%$, and $20 \%$ of the non-bridging $\mathrm{O}$ atoms protonated (referred to as HD_Y or LD_Y where $\mathrm{Y}$ indicates degree of protonation, respectively). The resulting surface density of $\mathrm{OH}$ groups is $13.6,6.8$, and $2.72 \mathrm{OH} / \mathrm{nm}^{2}$ for HD_100, HD_50, and HD_20, and 4.5, 2.25, and $0.90 \mathrm{OH} / \mathrm{nm}^{2}$ for LD_100, LD_50, and LD_20 surfaces, respectively. 
In our simulations the available surface density for SDS molecules on both HD and LD silica surfaces is $\sim 48.0 \AA^{2}$ per molecule, whereas for $\mathrm{C}_{12} \mathrm{E}_{6}$ on $\mathrm{LD}$ silica surfaces the surface density is $\sim 54.0 \AA^{2}$ per molecule.

The Groningen Machine for Chemical Simulations (GROMACS) simulation package, version 4.0.5, was used to integrate the equations of motion. ${ }^{25-26}$ The SPC/E model was used to represent water molecules. This model was used consistently in our previous publications and results in accurate description of many structural properties of water. ${ }^{22,}{ }^{27}$ The SPC/E model has some deficiencies, in particular in the estimation of the water vapor pressure, but yields reliable estimates for structure and dynamics of bulk water, in terms of radial distribution function and self-diffusion coefficient. Because the emphasis here is on the structural properties of water, this model should provide reliable insights. Because we focus on interfaces, polarizability effects could be important. However, in a recent report we showed that predictions regarding the structure of water near graphitic substrates do not depend strongly on the polarizability of the water model. ${ }^{40}$ Model parameters used for $\mathrm{C}_{12} \mathrm{E}_{6}$ and SDS molecules are described elsewhere. ${ }^{24}$, ${ }^{28}$ In particular, for only the 100_GRA system (SDS on graphene with $100 \AA^{2}$ of surface available per surfactant) we conducted additional simulations in which the point charges on the SDS head group atoms were modulated.

Dispersive forces were treated with an inner cutoff of $0.8 \mathrm{~nm}$ and outer cutoff of $1.0 \mathrm{~nm}$. Long range electrostatic interactions farther than $1.0 \mathrm{~nm}$ were treated using the particle mesh Ewald (PME) method with Fourier grid spacing of $0.12 \mathrm{~nm}$, tolerance of $1 \times 10^{-5}$, and fourth order interpolation as implemented in GROMACS. Three dimensional periodic boundary conditions were used for all the simulations. To reduce the un-physical interactions between periodic replicas of the water film along the $\mathrm{Z}$ direction a vacuum of size, at least, twice the thickness of 
the water film was introduced between the periodic replicas, following prior procedures in our group. In addition, the results were validated by considering the $2 \mathrm{D}$ pseudo summation PME algorithm, as implemented in GROMACS. ${ }^{25-26}$

The canonical ensemble, in which the number of particles $(\mathrm{N})$, the box volume $(\mathrm{V})$ and the temperature (T) were kept constant is used to perform all the simulations. Two substrates separated by more than $10 \mathrm{~nm}$ of vacuum, or one substrate separated from its image by more than $15 \mathrm{~nm}$ were used in our simulations. When two substrates are used, the water molecules fill the region between the substrates. The system temperature was maintained constant using the Nose-Hoover thermostat with a relaxation time of $100 \mathrm{fs}$ at $\mathrm{T}=300 \mathrm{~K}$. All the simulations on silica surfaces were at least $45 \mathrm{~ns}$ long, and trajectory data from last $5 \mathrm{~ns}$ were used to perform analysis. Simulations of SDS on graphite were at least $20 \mathrm{~ns}$ long and the last $2 \mathrm{~ns}$ were used for data analysis. The initial configuration for the simulations are equilibrated geometries borrowed from earlier publications from our group. ${ }^{29}$

\section{Results}

\subsection{Density, Orientation, and Density Fluctuations of Interfacial Water Molecules}

Surfactants. Before discussing interfacial water, we briefly summarize the results obtained for surfactants adsorbed on the silica surfaces. Full details are provided elsewhere. ${ }^{24}$ On the HD_100 silica surface, we observe multi-layer SDS adsorption. The first layer of SDS molecules adsorb parallel to the surface due to the electrostatic interactions between the hydroxyl groups on silica and the surfactant head groups; note that the methyl tail groups are perfectly aligned between the hydroxyl groups. ${ }^{24}$ The surface concentration of SDS molecules on the HD_100 silica surface is less than the monolayer coverage, as few SDS molecules diffuse to the bulk water and to the 
water-vacuum interface. Since HD_50 and HD_20 have non-protonated, negatively charged, oxygen atoms, the sodium counter-ions adsorb on the surface, near the non-bridging oxygen atoms, providing adsorption sites for the SDS head groups. On LD surfaces, irrespectively of the degree of protonation, SDS does not adsorb, but forms aqueous micellar aggregates near the silica-water interface. Even on the completely protonated LD_100 we observe less than five SDS molecules adsorbed onto the surface. As opposed to SDS, $\mathrm{C}_{12} \mathrm{E}_{6}$ adsorbs on the LD_20 surface with its ethylene oxide head groups on the surface, on LD_50 surface with either ethylene oxide or alkyl tail groups on the surface, and on the LD_100 surface mostly with alkyl tail groups on the surface.

Water. Density distributions and average $\mathrm{z}$-component dipole moment $\left(\mathrm{M}_{\mathrm{z}}\right)$ of water molecules as a function of the distance from the substrate serve to quantify surface effects on the packing and orientation of water molecules, respectively. These two quantities are reported in Figure 1 for water molecules next to the various silica surfaces in the presence of either ionic or non-ionic surfactants. For comparison, in the insets of Figure 1, we show the analogous results for water molecules on HD and LD silica substrates in the absence of surfactants, as reported in Ref [30]. The dipole moment vector of a water molecule points from the mid-point between the hydrogen atoms towards the oxygen. $\mathrm{M}_{\mathrm{z}}$ fluctuates around zero when the orientation of water molecules is isotropic. A positive $\mathrm{M}_{\mathrm{z}}$ corresponds to hydrogen atoms of water molecules oriented towards the surface, and negative $\mathrm{M}_{\mathrm{z}}$ indicates that water molecules orient their hydrogen atoms away from the surface. The surface coverage of SDS and $\mathrm{C}_{12} \mathrm{E}_{6}$ surfactants on the silica surfaces considered is $\sim 2.0$ surfactants $/ \mathrm{nm}^{2}$ and $\sim 1.8$ surfactants $/ \mathrm{nm}^{2}$, respectively.

On bare HD_100, in the absence of surfactants, we observe two intense peaks in the density profile of water molecules (solid line in the inset of the top-left panel of Figure 1) corresponding 
to a large number of interfacial water molecules accumulated near this surface. The intensity of the first peak in the density profiles for water on LD_100 (dotted line in the inset of the top-left panel) is less than that observed on HD_100 even though both surfaces show macroscopic hydrophilic features. ${ }^{31}$ Other properties of interfacial water on HD and LD silica have been described elsewhere. ${ }^{30,32}$ We focus here on the properties of interfacial water in the presence of counter-ions and surfactants. On the top-left panel of Figure 1 we report the density profiles in the presence of SDS (on HD_100, LD_100) and $\mathrm{C}_{12} \mathrm{E}_{6}$ (only on LD_100). The partial adsorption of SDS on HD_100 silica decreases by $\sim 16 \%$ and by $~ 40 \%$ the intensity of the first and second peaks in the density profiles of water on the bare HD_100. Despite these large changes in intensity, one important observation is that the location of the peaks in the density profiles does not change with the increased SDS concentration. This suggests that SDS and water compete to adsorb on HD_100, and that the water-surface interactions are not subdued by the lateral presence of surfactant molecules. Owing to the strong electrostatic interactions between negatively charged sulfate head group and surface hydroxyl atoms, SDS adsorbs on the substrate and replaces, in part, water. A depletion of water molecules is observed from $5.0 \AA$ to $25.0 \AA$ from the surface due to the presence of the tail groups of SDS. This is a consequence of steric effects. At larger distances the density of interfacial water approaches bulk values, suggesting that the presence of the surfactants affects the density of interfacial water only at short distances. The presence of SDS on the LD_100 decreases the intensity of the first and second peaks in the water density profile by $\sim 30 \%$ and $\sim 50 \%$, respectively, because of the adsorbed SDS molecules. Contrary to what observed on the HD surfaces, our results show that SDS adsorption on this surface affects also the peak positions in the water density profile, indicating the extended influence of SDS on the water molecules on this surface and that water-surface interactions are 
relatively less attractive for LD than HD surfaces. Indeed, we observe that on the LD surfaces water molecules are displaced from the interfacial region, the number of displaced molecules increasing with increasing degree of protonation of the non-bridging $\mathrm{O}$ atoms, due to the presence of surfactants and counter-ions.

Surprisingly, we found that the density of water molecules near LD silica surfaces in the presence of $\mathrm{C}_{12} \mathrm{E}_{6}$ surfactants, which adsorb significantly on the surface, is minimal on the LD_100 surface and increases as the degree of protonation decreases. This happens due to geometric constraints and electrostatic interactions between ethylene glycol head groups and the surface hydroxyl groups, as described elsewhere. ${ }^{24}$

Comparing the dipole moment orientation of water molecules on HD_100 surface in the presence and absence of SDS molecules, solid line in bottom-left panel of Figure 1, we observe an insignificant decrease in the intensity of the first peak in $\mathrm{M}_{\mathrm{z}}$ due to the presence of SDS, suggesting that the orientation of water molecules within $\sim 6.0 \AA$ from the surface does not change much. On the contrary, on the LD_100 surface water molecules in the first layer change significantly their orientation upon SDS adsorption. The hydrogen atoms pointing away from the surface observed on the bare LD_100 surface ( at 3.0-4.0 $\AA$ ) point preferentially towards the surface when SDS is present (this is likely a consequence of the water hydrogen atoms interacting with SDS head groups and counter-ions near the surface). The effect on water orientation is more pronounced on LD than HD silica surfaces because the density of nonbridging $\mathrm{O}$ atoms is much lower on the former than on the latter (4.5 vs. 13.6 per $\mathrm{nm}^{2}$ ) and therefore even a few SDS and/or counter-ions present at the interface affect strongly the behavior of interfacial water. Note however that patches of ordered water molecules can be observed away from surfactant head groups. 
Protonation

$(100 \%)$

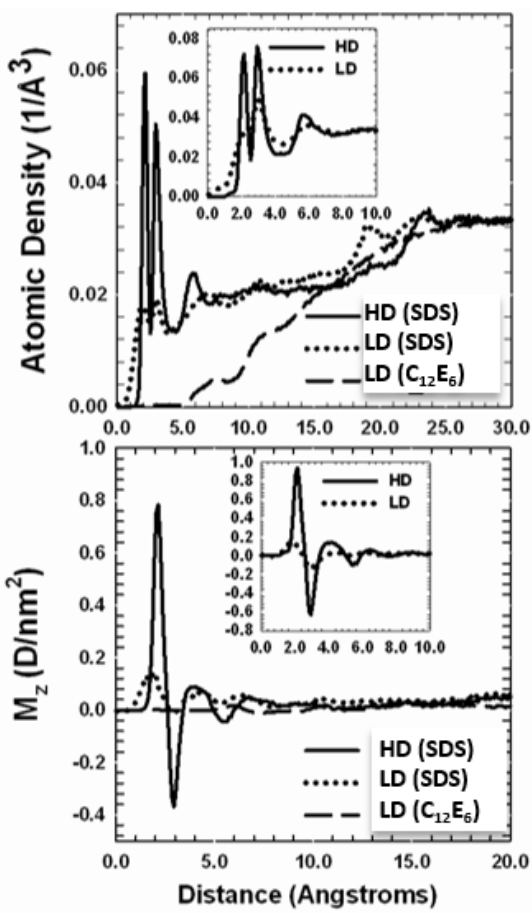

$(50 \%)$
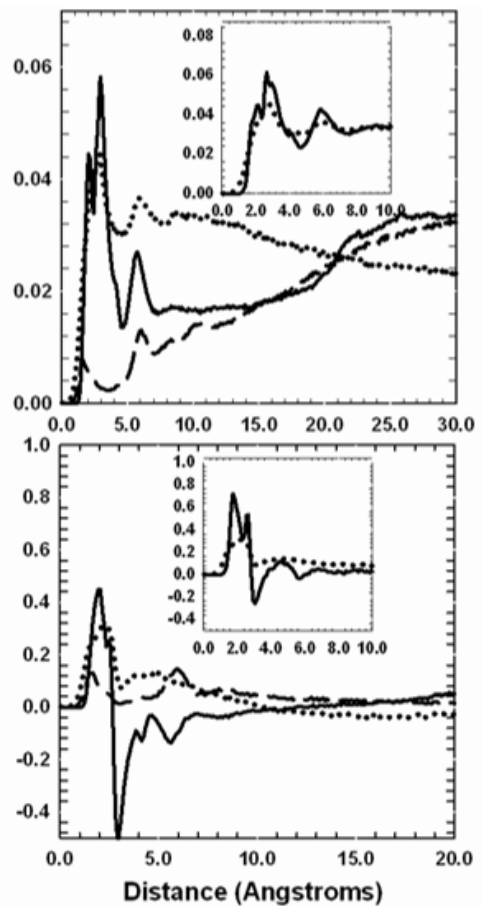

$(20 \%)$
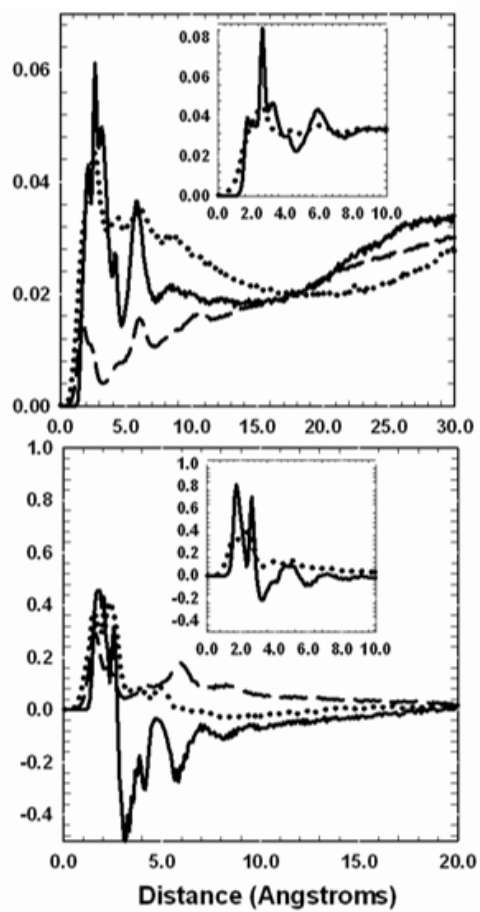

Figure 1: (Top panels) Density profiles of water molecules along the direction perpendicular to the solid HD and LD silica surfaces in the presence of adsorbed SDS and $\mathrm{C}_{12} \mathrm{E}_{6}$. Shown in the inset are the density profiles of water molecules on bare HD and LD silica surfaces. (Bottom panels) z-component of the dipole moment vector, normalized over the surface area, for interfacial water molecules as a function of distance from HD and LD silica in the presence of adsorbed SDS and $\mathrm{C}_{12} \mathrm{E}_{6}$. Shown in the insets are the normalized z-component dipole moments of water molecules at HD silica-water and LD silica-water interfaces with no surfactants present. The left panels are for $100 \%$ protonated, middle panels for $50 \%$ protonated and right panels for $20 \%$ protonated HD and LD silica surfaces, respectively.

On both HD_50 and HD_20 silica surfaces, the density of water in the first layer does not show significant changes upon SDS adsorption. The density differences farther away from the surfaces are due to steric effects correlated to the presence of SDS. However, in terms of the water orientation, we observe a greater than $25 \%$ decrease in the preference of the hydrogen atoms in the first layer to point towards the surface (bottom panel of Figure 1). The $\mathrm{M}_{\mathrm{z}}$ results suggest that the percent of the water hydrogen atoms pointing away from the surface increases considerably in the second water layer upon SDS adsorption. These structural effects are not 
observed for distances greater than $10 \AA$, which is consistent with recent reports regarding the interfacial water near discretely charged surfaces. ${ }^{33}$

SDS molecules do not adsorb on LD_50 and LD_20 surfaces and hence we do not observe any significant changes on the structure of interfacial water molecules on these substrates. At $\sim 10.0$ to $\sim 25.0 \AA$ away from the LD_50 surface, we observe a decrease in the density profiles, which is due to the presence of SDS aggregates in this region. Upon $\mathrm{C}_{12} \mathrm{E}_{6}$ adsorption, both density and orientation of interfacial water change. Specifically, the density of water molecules near the interface $(\sim 2-3 \AA)$ increases with decreasing hydroxyl density and these water molecules predominantly orient their hydrogen atoms towards the surface. Our results suggest that ethylene oxide groups and water molecules compete for interfacial spots on the LD_20 surface much more strongly than they do on the LD_100 surface.

In Figure 2 we plot the density profiles (right panel) and $\mathrm{M}_{\mathrm{z}}$ (left panel) for interfacial water near graphite. On this surface SDS yields a monolayer at surface coverage of $100 \AA^{2} /$ surfactant, a monolayer with patches of bilayers and hemi-cylinders at $60 \AA^{2} /$ surfactant, and a complete hemicylinder at $40 \AA^{2} /$ surfactant. $^{29}$ In the absence of surfactants (green dash-dot-dot line), we observe an intense peak in the water density profile at $\sim 3.4 \AA$, position at which corresponds a maximum in $\mathrm{M}_{\mathrm{z}}$. This indicates that most of the water molecules whose oxygen atoms are at $\sim 3.4$ $\AA$ from the surface adopt an orientation in which one hydrogen points towards the surface. This result is consistent with those reported in the literature. ${ }^{22,34-35}$ When the SDS surfactants adsorb on graphite, both the density and the orientation of interfacial water molecules change. At SDS surface coverage near $100.0 \AA^{2}$ per surfactant, we observe a density peak for water oxygen atoms at $\sim 3.3 \AA$ (solid line in the right panel of Figure 2 ), although the overall density at the interface is much less than that observed in the absence of the surfactants. The intensity of this peak 
decreases as the surfactant concentration increases, because as the SDS molecules are adsorbed water molecules are expelled from the interfacial region due to steric effects.
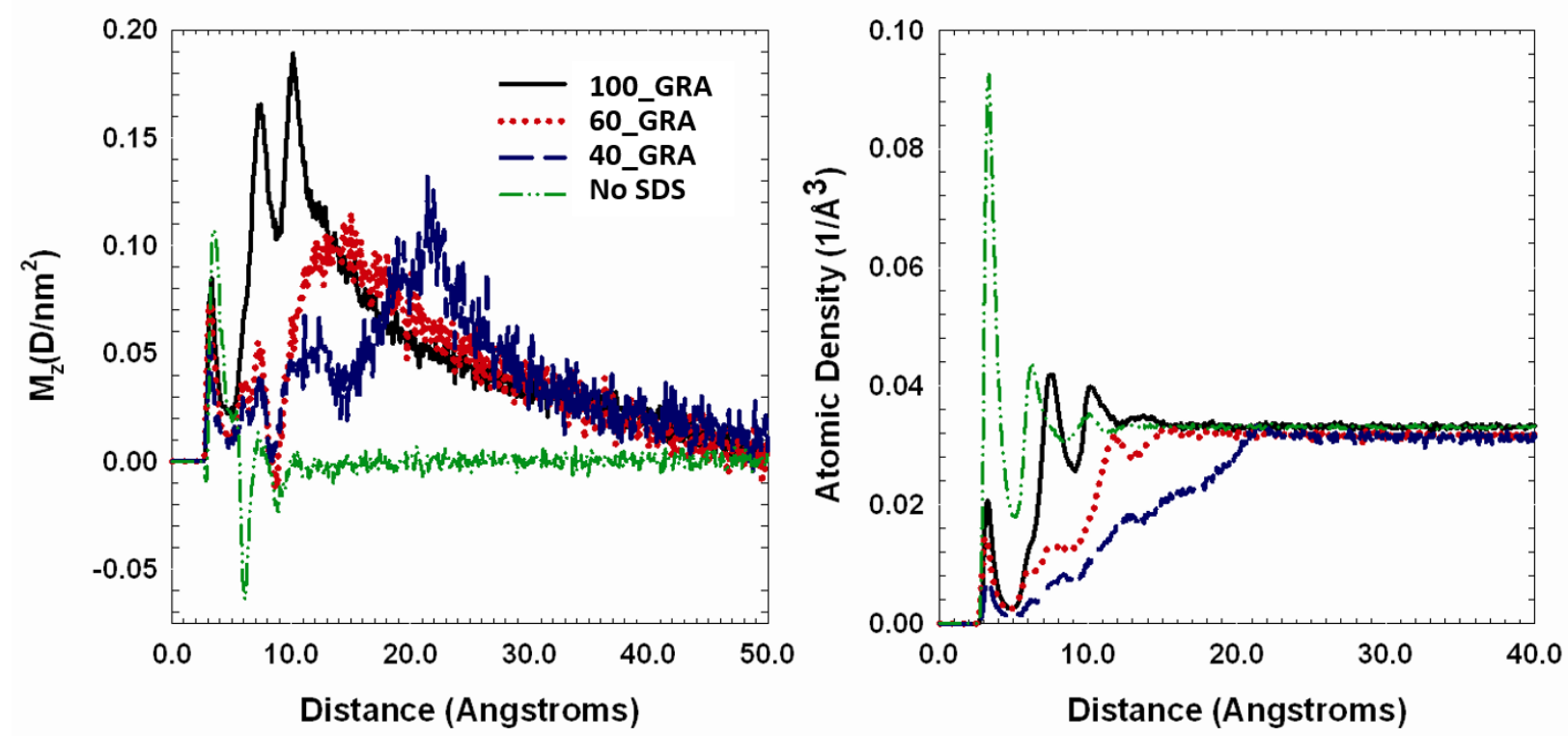

Figure 2: (Left panel) z-component of dipole moment vector of water molecules as a function of the distance perpendicular from graphite. (Right panel) Density profiles of oxygen atoms of water molecules from graphite. Results are shown at increasing concentration of SDS. X_GRA corresponds to graphite surface with $\mathrm{X} \AA^{2}$ available surface area per surfactant.

The presence of SDS changes the orientational profile of interfacial water. In the absence of SDS, we observe structured (alternate layers of hydrogen-down followed by hydrogen-up up to $\sim 10.0 \AA$ ) layers of interfacial water molecules (green dash-dot-dot line in the left panel of Figure 2). When SDS surfactants are present we do not observe peaks of hydrogen-up orientation (black, red, and blue lines). Since SDS surfactants are anionic, the positive peaks in $\mathrm{M}_{\mathrm{z}}$ correspond to layers of water that form next to the SDS head groups. These layers are displaced away from the graphite surface as the SDS surface concentration increases, because as the SDS aggregate morphology changes the location of SDS head groups with respect to the surface also 
changes, and water molecules are strongly correlated to the SDS head groups. From $\mathrm{M}_{z}$, we observe that interfacial water molecules show an increased hydrogen-down orientation for distances up to $5.0 \mathrm{~nm}$ away from graphite when SDS surfactants are present. This result is more impressive when we notice that in the absence of SDS water recovers bulk-like orientational features at 1.0-1.5 $\mathrm{nm}$ from the graphite. Similar orientational effects were observed for water in the presence of anionic and cationic surfactants at the air/water interface. ${ }^{36-37}$ The presence of SDS head groups on graphite effectively renders the surface partially charged, and charged surfaces have long-range effects on the orientation of water and dipolar liquids. ${ }^{38-39}$ Even though the peaks in density profiles cannot be used as signature for discriminating the surface features, ${ }^{7}$ the change in the density profiles due to surfactant adsorption is a microscopic feature that could be used to quantify how water-surface interactions are modified in presence of surfactants. In support of this argument, we refer to a prior simulation from our group, in which we found that SDS aggregates on graphite affect the morphology of SDS aggregates adsorbed on a parallel graphite surface placed at distances closer than $10.0 \mathrm{~nm} .^{23}$ From the density profiles (Figure 2, right panel), we observe that at the lowest SDS surface coverage considered here the density of water molecules reach bulk values at $2.0 \mathrm{~nm}$ from the surface. Increasing the surfactants concentration until they form hemi-cylinders (40_GRA), the effect of surfactants on the orientation of water is observed up to $\sim 3.0 \mathrm{~nm}$ away from the surface (because the head groups farthest from the graphite surface are at $\sim 2.0 \mathrm{~nm}$ from it). This orientational effect is of much longer range than that observed for pristine graphite. It is worth pointing out that similar effects were not observed on the silica substrates. 

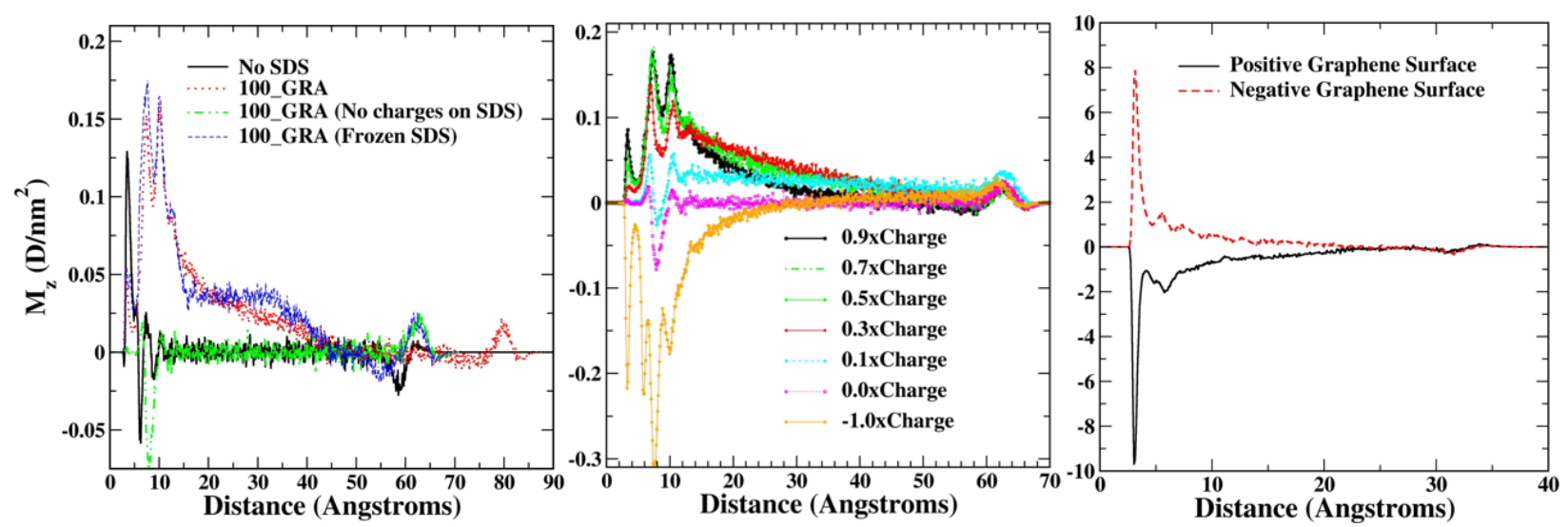

Figure 3: Average z-component dipole moment of water molecules as a function of the vertical distance from graphite (left). Comparing the effect of frozen SDS coordinates on orientational distribution of water away from the surface. Red dotted line corresponds to 100_GRA system with increased number of water molecules (middle). The charge on the model surfactant head groups is modulated from 0.1 to -1.0 times the charges of SDS (right). The results on the right panel are for $+/-10 \mu \mathrm{C} / \mathrm{m}^{2}$ uniformly charged graphene surfaces, from Ref. $\left[{ }^{40}\right]$, in which no surfactant is present. The peaks observed at $\sim 6 \mathrm{~nm}$ on the left panel and at $\sim 3-3.5 \mathrm{~nm}$ on the right panel are due to the presence of the liquid-vacuum interface.

We performed simulations for model systems to better understand the molecular driving forces responsible for the long-range perturbation in the ordering of interfacial water just discussed. We conducted two simulations: one with the force fields used in the prior simulations but fixing the coordinates of the SDS molecules adsorbed on the surface, and another one in which we set all the partial charges on SDS atoms and sodium counter-ions equal to zero (in the latter simulation the surfactants are allowed to move). The results obtained for $\mathrm{M}_{\mathrm{z}}$ at the water-graphite interface are shown in Figure 3 (left panel). The results unequivocally confirm that the partial charges on SDS and counter-ions provide the necessary interaction energy to partially order interfacial water. However, even freezing the SDS molecules adsorbed on the graphite surface is sufficient to affect the water orientation. Comparing the results obtained when the SDS molecules are free to move or frozen (while maintaining their partial charges) suggests that the 
fluctuations, due to thermal motion, occurring in the position of the charged species, i.e., the sulfate head groups, generate an approximately exponential decay in $\mathrm{M}_{\mathrm{z}}$ for water molecules away from the SDS head groups. When the model surfactants bear no partial charges, interfacial water regains isotropic orientation at distances lower than $1.5 \mathrm{~nm}$ from the surface, as previously reported. ${ }^{22}$ Our results therefore confirm that charged surfaces affect the structure of interfacial water. At charged SDS-graphite interface, the reasons for ordering of water molecules can be explained with the buildup of negative charges due to monolayer SDS adsorption on graphite, resulting in a discretely charged negative surface. It has been reported that negative surface charges cause water molecules ${ }^{23,29}$ to lose orientational entropy (in qualitative agreement with experimental observations) $)^{9,41}$ while enhancing the favorable electrostatic interactions. ${ }^{42-43}$ In addition, our results suggest that discrete charge distribution, and fluctuations in this distribution due to thermal motion yield longer-ranged effects on the orientation of interfacial water.

Additional simulations where conducted maintaining fixed the surfactants adsorbed on graphite, but modulating the partial charges from 0.1 to 0.9 times the values of the SDS parameterization and also reversing these charges (in these simulations the charges of the counter-ions were also modulated to maintain over-all electroneutrality). The results are shown in Figure 3. When the partial charges are 0.1 the original ones, the results for $\mathrm{M}_{\mathrm{z}}$ are similar to those obtained when the surfactants bear no charges. When the factor increases to 0.3 , we find that water molecules are oriented with their hydrogen atoms pointed away from the substrate at distances as large as $~ 5.0$ $\mathrm{nm}$. This result indicates that even when the individual charges are not pronounced, their accumulation due to surfactants aggregation can affect the orientation of interfacial water molecules. It is worth pointing out that the $M_{z}$ results obtained in the presence the model surfactants just described are comparable to those obtained for interfacial water near a uniformly 
charged surface (right panel of Figure 3). ${ }^{33,40}$ We also find that when the charges are reversed the water molecules recover their anisotropic orientation at about $30 \AA$ from the surface, which corresponds to $\sim 15-20 \AA$ from the surfactant head groups. This distance is similar to that at which interfacial water molecules recover their isotropic orientation near a uniformly charged graphene surface, $\sim 20 \AA$. Thus our results suggest that negatively charged surfactants have a stronger effect than positively charged ones on the orientation of interfacial water. The asymmetric nature of charge effects could be due to a lower entropy loss experienced upon preferential water re-orientation near a negative than near a positive charged head group, and it could also be due to the electrostatic interactions between the point charges due to the geometry of the water molecule. Our results are supported by the experimental observation that at air/water interfaces SDS affects the orientation of interfacial water beyond the first interfacial water layer, ${ }^{9}$ which indicates that at hydrophobic interfaces the effects on water orientation can be pronounced and long ranged.

In Figure 4 we report the contour plots of water $M_{z}$ in $X Y$-planes at 3 distances from the solid substrate, i.e., at $3.25 \AA, 10.25 \AA$, and $30.25 \AA$. We compare the results with those obtained for the pristine graphite-water interface. At $3.25 \AA$, on both surfaces, where water is present we observe preferential hydrogen-down orientation. At $10 \AA$ the preferential hydrogen-down orientation is pronounced when SDS is present while isotropic orientation prevails on pristine graphite. On the latter substrate we observe no significant difference between the contour plots obtained at 10.25 and $30.25 \AA$. In the presence of SDS even at $30.25 \AA$ we observe patches of hydrogen-down orientation, indicating that, even though water orientation is almost isotropic, the orientation is not uniform within the XY-plane and some water molecules show preferred orientation. These results suggest that interfacial water could yield extended structures along the 
direction normal to the surfaces because of the presence of the surfactants. Such structures could influence proton or ion transport similarly to the enhanced ion transport in columnar/cylindrical structures. ${ }^{44}$ In fact, the Widom insertion free energy of an $0.3 \mathrm{~nm}$ cation at $\sim 3.0 \mathrm{~nm}$ away from the graphite in presence of SDS surfactants (in a radius of $0.3 \mathrm{~nm}$ at $x=5.0$ and $y=4.0 \mathrm{~nm}$ in the top left panel of Figure 3) is, on an average, $\sim 50 \mathrm{~kJ} \mathrm{~mol}^{-1} \mathrm{~nm}^{-3}$ more favorable than that computed in the same location in the absence of SDS surfactants.
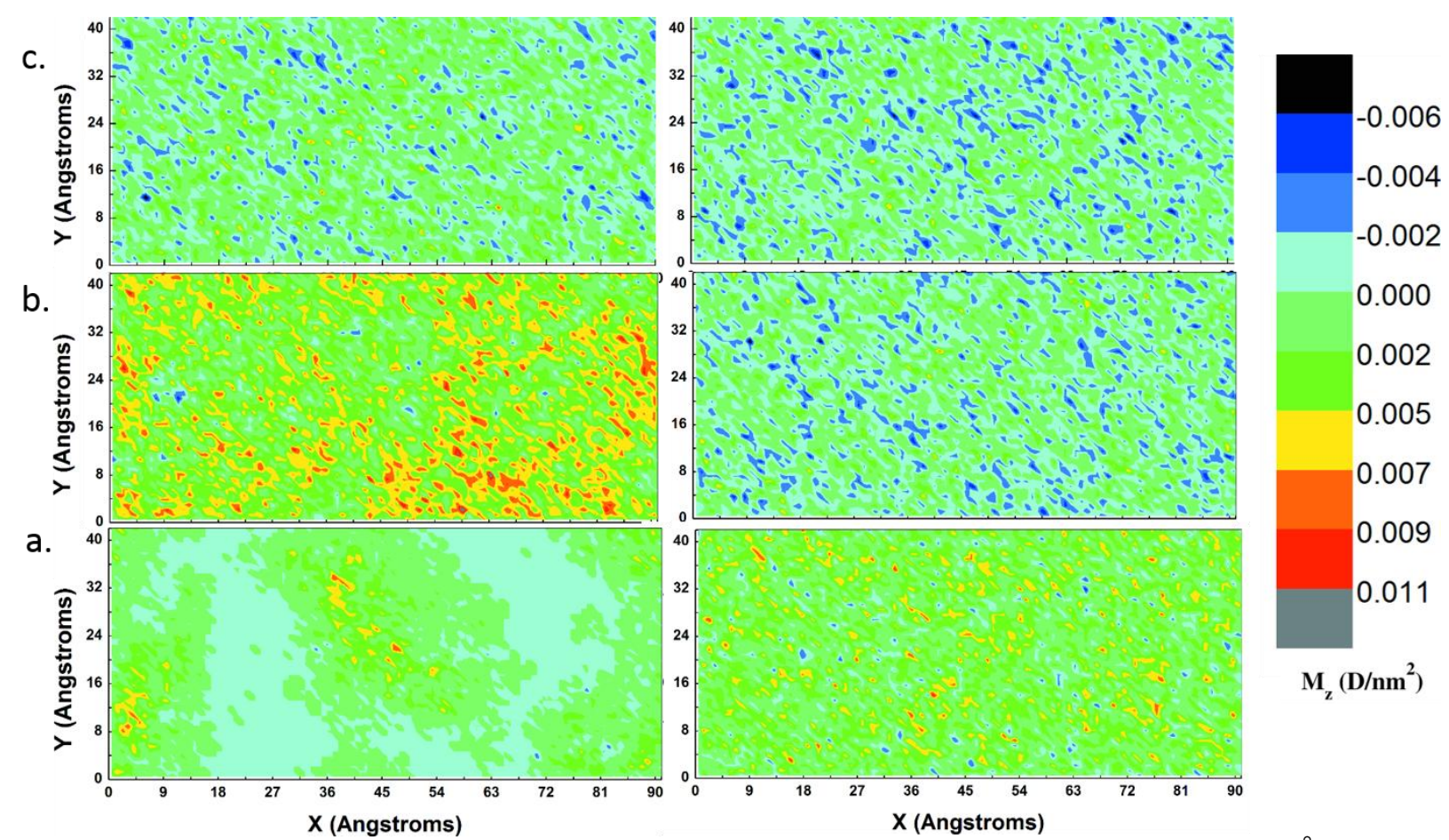

Figure 4: Contour plots of $\mathrm{Mz}$ calculated for water molecules within a slab of $0.75 \AA$ located between a.) $3.25-4.0 \AA$, b.) $10.25-11 \AA$, and c.) $30.25-31 \AA$ from the substrate. Left and right panels are for the water molecules in the graphite-water interface with and without SDS, respectively. For the water density at the various distances away from graphite surface please refer to the right panel of Figure 2.

Surprisingly, the ordering of interfacial water molecules does not couple with changes in density fluctuations (which can provide important details regarding surface properties ${ }^{7}$ ). We report as Supplemental Information the water isothermal compressibility computed from density 
fluctuations near various interfaces. Our results indicate bulk water compressibility of $\sim 60 \times 10^{-11}$ $\mathrm{Pa}^{-1}$, which is slightly larger than the experimental value of $\sim 45 \times 10^{-11} \mathrm{~Pa}^{-1} \cdot{ }^{45}$ Large density fluctuations are observed close to the graphite surface with or without SDS present. Owing to these large density fluctuations, our results indicate that it is more probable to fit a cavity at the graphite-water at distances lower than $3.3 \AA$ than near either HD or LD silica surfaces, whether or not SDS is present. This analysis corroborates the results obtained by Patel et al., ${ }^{46}$ in terms of the cavity formation at hydrophilic and hydrophobic interfaces.

We also computed the excess chemical potential ( $\left.\mu_{\text {excess }}\right)$ of neutral, anionic, and cationic spheres of size 0.3 and $0.35 \mathrm{~nm}$ within the interfacial region, using the Widom insertion method. ${ }^{47}$ The details are presented as Supplemental Information. Within water layers away from pristine graphite surface the $\mu_{\text {excess }}$ for a neutral sphere is always positive, while inserting anions of diameter 0.3 and $0.35 \mathrm{~nm}$ is favorable by $\sim 180$ and $\sim 135\left(\mathrm{~kJ} \mathrm{~mol}^{-1}\right)$, respectively. Strong fluctuations are observed for the excess chemical potential when inserting anions of diameter $0.30 \mathrm{~nm}$ in presence of SDS. At $\sim 1.5 \mathrm{~nm}$ away from the surface the minimum excess chemical potential is of $\sim 220 \mathrm{~kJ} \mathrm{~mol}^{-1}$, compared to $\sim 180 \mathrm{~kJ} \mathrm{~mol}^{-1}$ in the absence of SDS at multiple distances from the surface. Note that that these values are averages obtained over the entire plane parallel to the surface, and that there can be flucutations due to the presence of SDS and sodium counter-ions at specific sites. The anions of diameter $0.30 \mathrm{~nm}$ can be inserted more favorably at distances larger than $4.0 \mathrm{~nm}$ from the surface, suggesting that the orientation of water molecules caused by the interface does affect the solvation of ions. Note that differences in solvation and excess chemical potential could cause changes in the diffusive properties of ions, although such detailed calculations have not been considered here. We found a notable, albeit small, difference in the excess chemical potential for cations due to the presence of SDS, while near silica the 
presence of SDS causes no difference in the calculated excess chemical potentials, which seems to agree with the little changes noted for the orientation of interfacial water at distances larger than $\sim 15 \AA$ from the surfactant head groups on this substrate. At short distances the surfactants have a clear effect on the chemical potential results.

\subsection{Residence Probability and Reorientational Dynamics}

We determined the residence probability function $(\mathrm{P})$ as the ratio of the number of water molecules present in the interfacial layer at time ' $t$ ' to the number of water molecules in the interfacial layer at time zero $(t=0)$. The longer the water molecules stay in a layer, the more slowly P decays. To assess the re-orientation dynamics of water molecules we computed the dipole-dipole auto-correlation function (DACF), defined as

$$
\mathrm{DACF}=\left\langle\frac{\mu_{w}(t) \mu_{w}(0)}{\mu_{w}(0) \mu_{w}(0)}\right\rangle
$$

DACF is 1.0 at time zero and decays gradually as the water molecules rotate. Water molecules re-entering into the interfacial layer or hydration shell are not considered in our P and DACF calculations. Considering molecules re-entering the hydration shell, within a very short time span, may bias the results towards slower-than-expected dynamics in comparison with experiments. ${ }^{48}$ The simulation results depend on how frequently the coordinates of water molecules are stored during the MD simulations, however because we used identical frequency of trajectory outputs, the results can be qualitatively compared against experiments.

We only consider water molecules within a distance of $5.1 \AA$ away from graphite and $3.3 \AA$ away from silica surfaces. These distances correspond to the position of the first minima in the water oxygen density profiles. Our calculations can also discriminate $\mathrm{P}$ and DACF for those water molecules that are simultaneously present in the interfacial layer and in the hydration shell 
of surfactants. To discriminate the water molecules within the first hydration shell around surfactants we use the cut-off distances of $5.15 \AA$ for SDS and $7.95 \AA$ for $\mathrm{C}_{12} \mathrm{E}_{6}$, as determined from the first minima in the sulfur (SDS) - oxygen (water) radial distribution function (RDF) and from the RDF between the center of mass of $\mathrm{C}_{12} \mathrm{E}_{6}$ head groups-oxygen (water), respectively. Literature results show that both translational and rotational dynamics of water molecules in the hydration shell of surfactant head groups and/or charged sites on a surface are slower than those in the bulk, ${ }^{49}$ although water reorientation depends on size and charge of the site/ion being hydrated $^{1,50}$ and also the polarity of the surface. ${ }^{51}$

In Figure 5, we report the results obtained for the residence probability $\mathrm{P}$ for water molecules near the silica surfaces without (inset of top panels) and with surfactants. $\mathrm{P}$ for water molecules on bare HD silica surfaces with varying degrees of protonation decays to $\sim 0.1$ in $400 \mathrm{ps,}$ although the curves are not identical. This suggests that the mechanism of water molecules movement from the first hydration layer varies with the density of surface $\mathrm{OH}$ groups. The anisotropic reorientation of water on these surfaces was discussed by Argyris et al. ${ }^{30}$

On HD surfaces, the presence of SDS causes the results for P obtained on the various surfaces to become very similar to each other, with 400 ps needed to decay to $\sim 0.2$ in all cases. On all LD surfaces, the presence of SDS delays the decay of P. On the LD surfaces, the presence of $\mathrm{C}_{12} \mathrm{E}_{6}$ yields a strong effect on $\mathrm{P}$, with the decay time of $\mathrm{P}$ directly proportional to the presence of $\mathrm{C}_{12} \mathrm{E}_{6}$ headgroups.

For water molecules simultaneously present in interfacial layer and hydration shell, multiple factors affect orientational and residence times: 1.) the presence of surfactant aggregates or individual surfactants at the surface (decays slower with the increase in aggregate size, as the diffusivity is inversely proportional to the aggregate size), and 2.) whether the tail groups or 
headgroups are close to the water molecules next to the surface. The presence of tail groups next to the surface inhibits longer residence times and vice-versa simply because of the competition between hydrophobic and electrostatic interactions. The P curves for water molecules within the hydration shell of surfactants are reported in the bottom panels of Figure 5. At short observation times the water molecules near $\mathrm{C}_{12} \mathrm{E}_{6}$ show the slowest decay in $\mathrm{P}$ due to the size of the ethylene glycol head group; at longer observation times the overall decay rate is similar to that observed for water in the presence of SDS on HD silica, because of the weaker attractions. Similarly, the P decay for water molecules in the hydration shells is dependent on the aggregate structure and size of the surfactants. When both surfactants are arranged as monolayers, the length of the headgroup is directly related to the mechanism of diffusion out of the hydration shell.

The direct comparison of the dynamics of water molecules next to surfactant head groups and next to immobile solid substrate is not straightforward, as the translation and rotation of surfactants molecules enhance the diffusion of water molecules within the surfactants hydration shell. On the contrary the rigidity of a solid substrate reduces the rate of translational and rotation dynamics of hydration water. ${ }^{30-31}$ However, comparing top and central panels of Figure 5, we observe that $\mathrm{P}$ for water molecules that are simultaneously present in both the interfacial and surfactant head groups hydration layers decays faster than that for water molecules only present in the surface hydration layers. This suggests that water molecules present in the first interfacial layer near the silica substrate and not near the surfactants contribute to the slow decay of $\mathrm{P}$, while water molecules within the hydration layer of surfactant head groups contribute to fast decay in P. These results are consistent with those reported for water in the hydration shell of surfactants. ${ }^{52}$ However, the residence times of interfacial water molecules depend on the 
magnitude of the discrete partial charge on the substrate ${ }^{53}$ and surface density of the charge for water in the hydration shell of ions. ${ }^{50}$
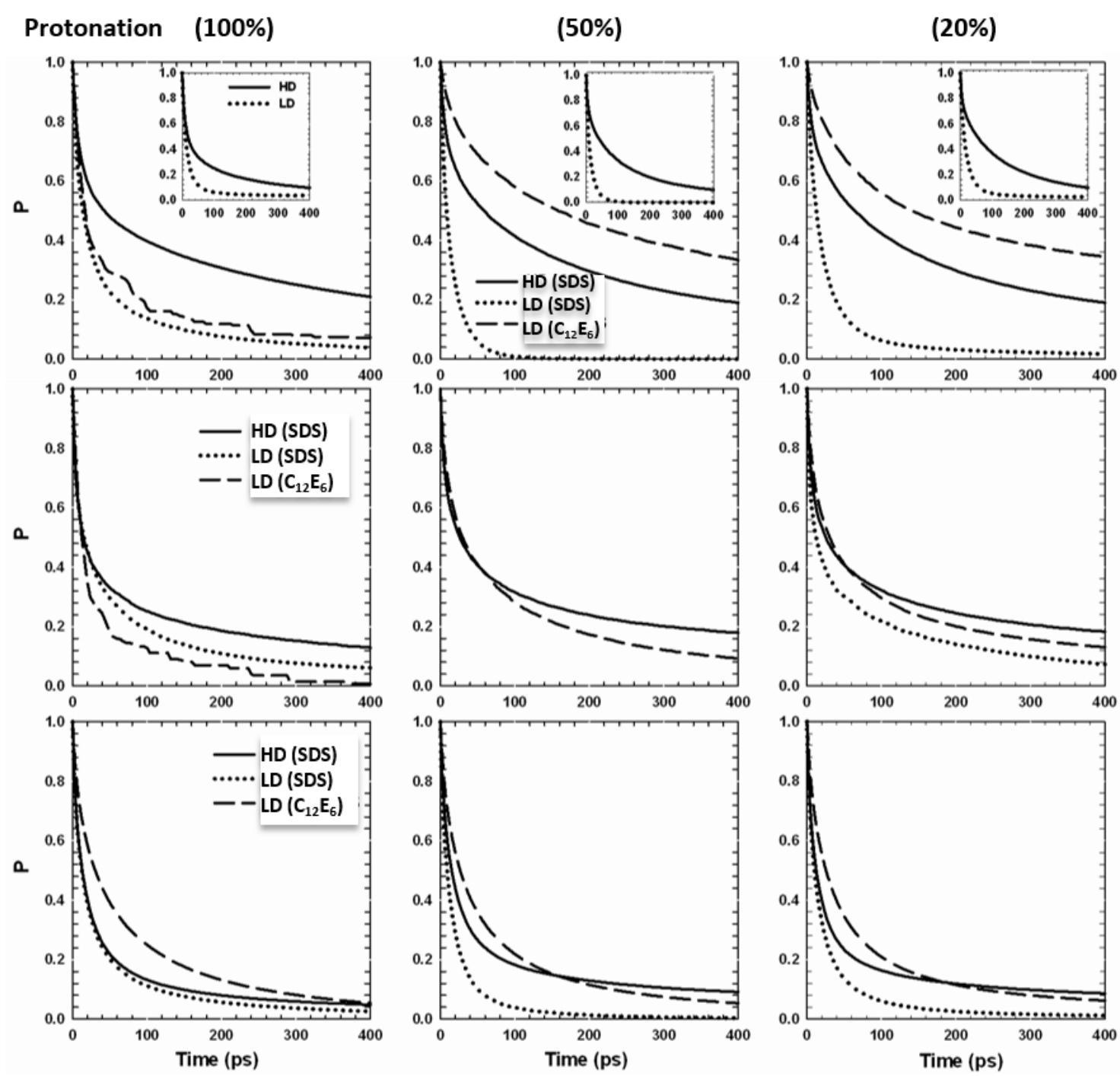

Figure 5: (Top panels) Residence probability function $(\mathrm{P})$ for water molecules in the interfacial layer of $\mathrm{HD}$ and LD silica surfaces in the presence of adsorbed SDS and $\mathrm{C}_{12} \mathrm{E}_{6}$. (Middle panels) $\mathrm{P}$ of water molecules that are simultaneously present in the interfacial layer and in the hydration shell of surfactants head groups on HD and LD silica surfaces in the presence of adsorbed SDS and $\mathrm{C}_{12} \mathrm{E}_{6}$. (Bottom panels) $\mathrm{P}$ of water molecules in the hydration shell of surfactants head groups on $\mathrm{HD}$ and LD silica surfaces in the presence of adsorbed SDS and $\mathrm{C}_{12} \mathrm{E}_{6}$. The left panels are for $100 \%$ protonated, middle panels for $50 \%$ protonated and right panels for $20 \%$ protonated HD and LD silica surfaces, respectively. 
In Figure 6, we show $\mathrm{P}$ and DACF for interfacial water molecules on graphite with and without SDS. We observe the fastest decay for P in the absence of SDS (dashed line in the left panel). The slowest decay is observed for water on 40_GRA_and 60_GRA, i.e., when SDS yields a hemicylindrical or multi-layered aggregate structure. As expected, when SDS surfactants form multiple layers or hemi-cylinders (60_GRA and 40_GRA, respectively), we observe that water molecules move out of the interfacial layer more slowly than observed on 100_GRA.
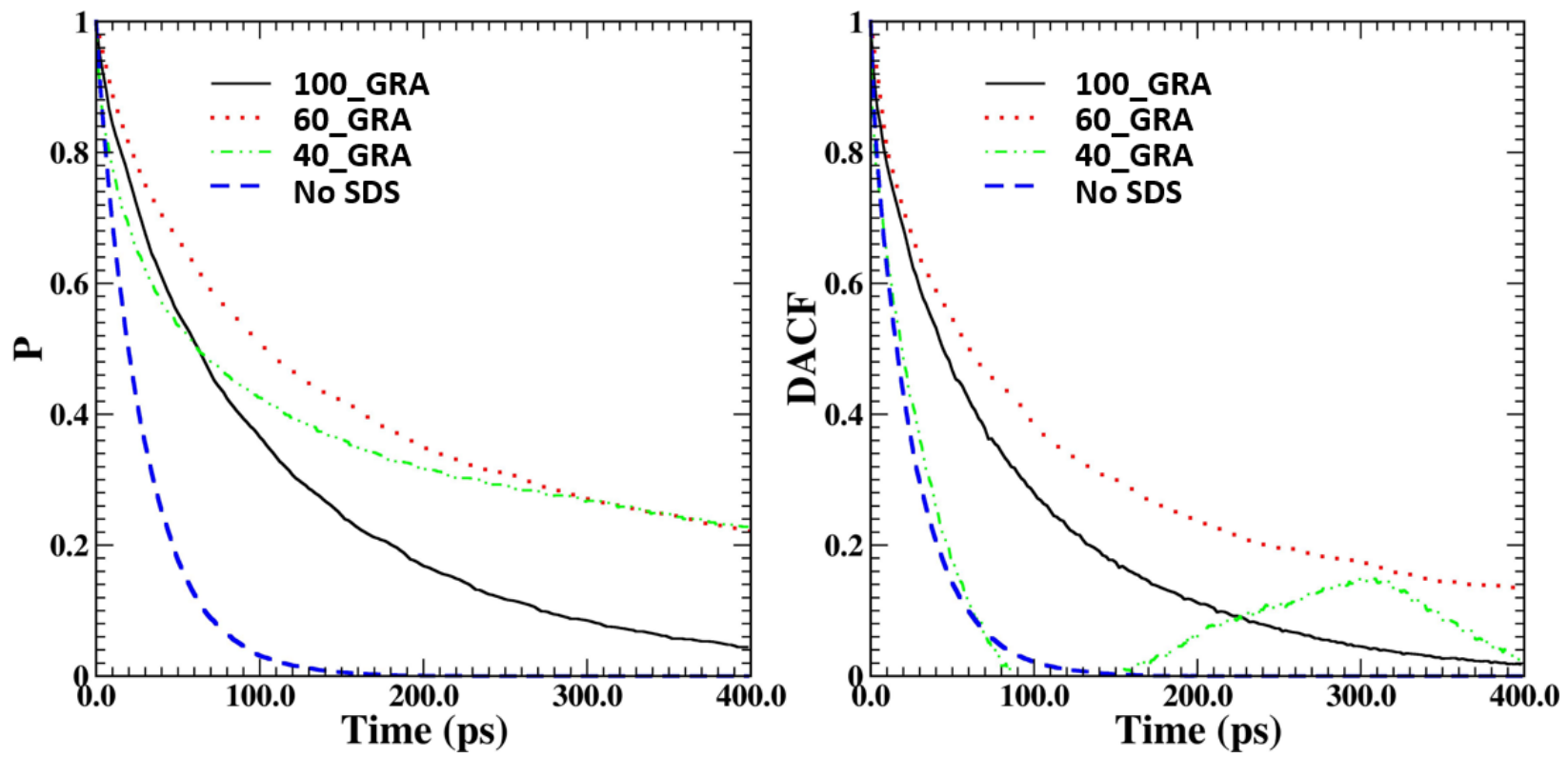

Figure 6: Left: Residence probability function $(\mathrm{P})$ for water molecules in the interfacial layer (within $5.1 \AA$ from graphite). Right: Dipole-dipole auto-correlation function (DACF) for interfacial water molecules.

While we observe that $\mathrm{P}$ is qualitatively similar for 40_GRA and 60_GRA, the DACF curves are different. This indicates that water molecules in the interfacial layer experience different environments on 60_GRA and 40_GRA. These local environments, which depend on neighboring moieties (head groups or tail groups) and on the diffusivity of individual surfactant molecules or aggregates, affect both translational and rotational diffusion of water molecules. 
For water molecules on bare graphite and on graphite with a hemi-cylindrical surface aggregate DACF curves decay at similar rates for the first $100.0 \mathrm{ps}$, suggesting that the water molecules on the 40_GRA system are in close proximity to the hydrophobic moieties (visual observation suggests that water molecules are in between the SDS hemicylindrical aggregates). More results for P and DACF for interfacial water molecules are shown in the Supplemental Information. Even though the water molecules next to ionic surfactant head groups ${ }^{5}$ reorient more slowly than in the bulk, the observed reorientation is faster than that observed for water confined in a hydrophobic liquid or reverse micelles. ${ }^{1,3,48}$ These results indicate that even though SDS surfactants induce long-ranged effects on the orientation of the water molecules away from the surface, at close proximity the effects of substrate on water residence times and orientational dynamics are stronger than those due to SDS head groups alone, probably because of the rigidity

of the substrate. We also found that the residence time of water molecules within slabs of increasing thickness away from the graphite surface $(1.0 \mathrm{~nm}, 2.0 \mathrm{~nm}$ and $3.0 \mathrm{~nm})$ are shorter when surfactants are present, indicating that SDS surfactants not only enhance the orientational order of the water molecules but also the diffusion away from the surface.

\section{Discussion \& Conclusions}

The analysis of the water next to anionic and nonionic surfactants adsorbed on model silica and graphite substrates sheds light on the combined effects of substrates and surfactants on packing, orientation and dynamic properties of interfacial water. In particular, our results provide fundamental understanding of some important effects due to the presence of surfactants.

Firstly, our results indicate that water molecules experience long-ranged orientational ordering especially near hydrophobic substrates covered with anionic surfactants, while the effects near hydrophilic silica surfaces are not very pronounced. Below we highlight and explain 
how such ordering affects hydration energy and the entropy of interfacial water. It has been documented how density fluctuations can be used to quantify the hydrophobicity of a substrate, ${ }^{54}$ and how such fluctuations, along with orientational ordering, contribute to the entropy of liquid water. ${ }^{55-56}$ The first term in computing the change in entropy of liquid water due to solute transfer results from density fluctuations at a few molecular shells away from the solute, ${ }^{57}$ followed by changes in orientational correlation. ${ }^{58}$ Because our results show rather similar density fluctuations for water at the graphite interface whether SDS surfactants are present or not, the orientational correlation of interfacial water becomes the dominant term in determining the entropy changes. Although the orientational correlation is multi-dimensional, using dipolemoment orientation we observe an increase in orientational order for interfacial water caused by the interface, which thereby decreases the loss of entropy upon the formation of a cavity in the water for distances up to at least $4.0 \mathrm{~nm}$ from the interface. The entropy loss and enthalpy gain due to the orientational ordering can also influence the diffusive properties of small molecules in water, which can potentially affect oxidative properties of many charged substrates, including substrates decorated with ions. The absence of such long-ranged water ordering near silica surfaces indicates that the electric field due to discrete charges distributed on the solid surface effectively reduces the effect of the long-range Columbic interactions due to anionic surfactants and ions adsorbed on the surface. This implies that on many substrates, not only the surface charges, but also the charges embedded within the first few atomic layers from the interface can play a significant role in determining interfacial water orientation, diffusion, and packing.

Our results from the analysis of the dynamic properties of interfacial water indicate that the water molecules close to surfactant head groups have faster dynamics even when close to the solid substrates, both in terms of residence lifetimes and reorientation dynamics, compared to 
that of water molecules within the solid-liquid interfacial layer and/or away from surfactant molecules (i.e., bulk water). The results discussed above indicate that surfactants at the watersolid interface can not only change the wetting characteristics of a surface, as previously

known, ${ }^{59-60}$ but also change the residence times of water in the interfacial regions, presumably by disrupting the local structure of interfacial water molecules. It is possible that these changes in interfacial water dynamics are relevant for tribological and geological properties, ion-exchange dynamics, enhanced mobility of dissolved gaseous molecules at interfaces, and perhaps even corrosion.

\section{Acknowledgements}

The authors acknowledge generous computing allocations at the Oklahoma Super-computing Center for Education \& Research (OSCER), Norman, OK, USA, and at National Energy Research Scientific Computing Center (NERSC), Berkeley, CA, USA.

Electronic Supporting Information (ESI) Available: Simulation results for compressibility of water computed from density fluctuations at pristine graphite and silica interfaces, at the interface of $\mathrm{C}_{12} \mathrm{E}_{6}$ and $\mathrm{LD}$ silica surfaces with varying levels of hydroxylation, at $100 \%$ protonated HD (13.6 OH/nm2) and LD (4.5 OH/nm2) surfaces in presence of SDS surfactant molecules, at graphite interface in presence of SDS surfactant molecules with varying surfactant concentration, excess chemical potential computed using Widom particle insertion method, residence times $(\mathrm{P})$, and dipole auto-correlation functions (DACF) of water molecules at various interfaces are shown in ESI. This information is available free of charge via the Internet at http://pubs.acs.org/

\section{References}


1. Fenn, E. E.; Wong, D. B.; Fayer, M. D. Water dynamics at neutral and ionic interfaces. Proc. Natl. Acad. Sci. USA 2009, 106, 15243-15248.

2. Moilanen, D. E.; Fenn, E. E.; Wong, D.; Fayer, M. D. Geometry and Nanolength Scales versus Interface Interactions: Water Dynamics in AOT Lamellar Structures and Reverse Micelles. J. Am. Chem. Soc. 2009, 131, 8318-8328.

3. Moilanen, D. E.; Levinger, N. E.; Spry, D. B.; Fayer, M. D. Confinement or the Nature of the Interface? Dynamics of Nanoscopic Water. J. Am. Chem. Soc. 2007, 129, 14311-14318.

4. Allen, H. C.; Casillas-Ituarte, N. N.; Sierra-Hernandez, M. R.; Chen, X.; Tang, C. Y. Shedding light on water structure at air-aqueous interfaces: ions, lipids, and hydration. Phys. Chem. Chem. Phys. 2009, 11, 5538-5549.

5. Chanda, J.; Bandyopadhyay, S. Molecular Dynamics Study of a Surfactant Monolayer Adsorbed at the Air/Water Interface. J. Chem. Thoery Comput. 2005, 1, 963-971.

6. $\quad$ Bruce, C. D.; Berkowitz, M. L.; Perera, L.; Forbes, M. D. E. Molecular Dynamics Simulation of Sodium Dodecyl Sulfate Micelle in Water: Micellar Structural Characteristics and Counterion Distribution. J. Phys. Chem. B 2002, 106, 3788-3793.

7. Godawat, R.; Jamadagni, S. N.; Garde, S. Characterizing hydrophobicity of interfaces by using cavity formation, solute binding, and water correlations. Proc. Natl. Acad. Sci. USA 2009, $106,15119-15124$.

8. Hummer, G.; Garde, S. Cavity Expulsion and Weak Dewetting of Hydrophobic Solutes in Water. Phys. Rev. Lett. 1998, 80, 4193.

9. Nihonyanagi, S.; Yamaguchi, S.; Tahara, T. Counterion Effect on Interfacial Water at Charged Interfaces and Its Relevance to the Hofmeister Series. J. Am. Chem. Soc. 2014, 136, 6155-6158.

10. Jungwirth, P.; Cremer, P. S. Beyond Hofmeister. Nat Chem 2014, 6, 261-263.

11. Qiao, B.-F.; Sega, M.; Holm, C. Properties of water in the interfacial region of a polyelectrolyte bilayer adsorbed onto a substrate studied by computer simulations. Phys. Chem. Chem. Phys. 2012, 14, 11425-11432.

12. Nihonyanagi, S.; Mondal, J. A.; Yamaguchi, S.; Tahara, T. Structure and Dynamics of Interfacial Water Studied by Heterodyne-Detected Vibrational Sum-Frequency Generation. Annu. Rev. Phys. Chem. 2013, 64, 579-603.

13. Anim-Danso, E.; Zhang, Y.; Dhinojwala, A. Freezing and Melting of Salt Hydrates Next to Solid Surfaces Probed by Infrared-Visible Sum Frequency Generation Spectroscopy. J. Am. Chem. Soc. 2013, 135, 8496-8499.

14. Scheu, R.; Chen, Y.; de Aguiar, H. B.; Rankin, B. M.; Ben-Amotz, D.; Roke, S. Specific Ion Effects in Amphiphile Hydration and Interface Stabilization. J. Am. Chem. Soc. 2014, 136, 2040-2047.

15. Gutig, C.; Grady, B. P.; Striolo, A. Experimental Studies on the Adsorption of Two Surfactants on Solid-Aqueous Interfaces: Adsorption Isotherms and Kinetics. Langmuir 2008, 24, 4806-4816.

16. Shi, L.; Ghezzi, M.; Caminati, G.; Lo Nostro, P.; Grady, B. P.; Striolo, A. Adsorption Isotherms of Aqueous $\mathrm{C}_{12} \mathrm{E}_{6}$ and Cetyltrimethylammonium Bromide Surfactants on Solid Surfaces in the Presence of Low Molecular Weight Coadsorbents. Langmuir 2009, 25, 55365544.

17. Lu, J. R.; Li, Z. X.; Thomas, R. K.; Staples, E. J.; Thompson, L.; Tucker, I.; Penfold, J. Neutron Reflection from a Layer of Monododecyl Octaethylene Glycol Adsorbed at the Air- 
Liquid Interface: The Structure of the Layer and the Effects of Temperature. J. Phys. Chem. 1994, 98, 6559-6567.

18. Lu, J. R.; Marrocco, A.; Su, T. J.; Thomas, R. K.; Penfold, J. Adsorption of Dodecyl Sulfate Surfactants with Monovalent Metal Counterions at the Air-Water Interface Studied by Neutron Reflection and Surface Tension. J. Colloid Interface Sci. 1993, 158, 303-316.

19. Penfold, J.; Tucker, I.; Thomas, R. K. Adsorption of Nonionic Surfactant Mixtures at the Hydrophilic Solid-Solution Interface. Langmuir 2005, 21, 6330-6336.

20. Purcell, I. P.; Thomas, R. K.; Penfold, J.; Howe, A. M. Adsorption of SDS and PVP at the air/water interface. Colloids and Surfaces A: Physicochemical and Engineering Aspects 1995, 94, 125-130.

21. Bandyopadhyay, S.; Chanda, J. Monolayer of Monododecyl Diethylene Glycol Surfactants Adsorbed at the Air/Water Interface: A Molecular Dynamics Study. Langmuir 2003, $19,10443-10448$.

22. Argyris, D.; Tummala, N. R.; Striolo, A.; Cole, D. R. Molecular Structure and Dynamics in Thin Water Films at the Silica and Graphite Surfaces. J. Phys. Chem. C 2008, 112, 1358713599.

23. Tummala, N. R.; Striolo, A. Role of Counterion Condensation in the Self-Assembly of SDS Surfactants at the Water-Graphite Interface. J. Phys. Chem. B 2008, 112, 1987-2000.

24. Tummala, N. R.; Shi, L.; Striolo, A. Molecular dynamics simulations of surfactants at the silica-water interface: Anionic vs nonionic headgroups. J. Colloid Interface Sci. 2011, 362, 135143.

25. Berendsen, H. J. C.; Vanderspoel, D.; Vandrunen, R. GROMACS - A Message PassingParallel Molecular-Dynamics Implementation. Comput. Phys. Commun. 1995, 91, 43-56.

26. Hess, B.; Kutzner, C.; van der Spoel, D.; Lindahl, E. GROMACS 4: Algorithms for highly efficient, load-balanced, and scalable molecular simulation. J. Chem. Thoery Comput. 2008, 4, 435-447.

27. Berendsen, H. J. C.; Grigera, J. R.; Straatsma, T. P. The missing term in effective pair potentials. J. Phys. Chem. 1987, 91, 6269-6271.

28. Shi, L.; Tummala, N. R.; Striolo, A. $\mathrm{C}_{12} \mathrm{E}_{6}$ and SDS Surfactants Simulated at the Vacuum-Water Interface. Langmuir 2010, 26, 5462-5474.

29. Tummala, N. R.; Striolo, A. Curvature effects on the adsorption of aqueous sodiumdodecyl-sulfate surfactants on carbonaceous substrates: Structural features and counterion dynamics. Physical Review E (Statistical, Nonlinear, and Soft Matter Physics) 2009, 80, 021408021410.

30. Argyris, D.; Cole, D. R.; Striolo, A. Dynamic Behavior of Interfacial Water at the Silica Surface. J. Phys. Chem. C 2009, 113, 19591-19600.

31. Ho, T. A.; Argyris, D.; Papavassiliou, D. V.; Lee, L. L.; Cole, D. R.; Striolo, A. Interfacial Water on Crystalline Silica:A Comparative Molecular Dynamics Simulation Study. Molecular Simulation 2010.

32. Argyris, D.; Cole, D. R.; Striolo, A. Hydration Structure on Crystalline Silica Substrates. Langmuir 2009, 25, 8025-8035.

33. Dewan, S.; Carnevale, V.; Bankura, A.; Eftekhari-Bafrooei, A.; Fiorin, G.; Klein, M. L.; Borguet, E. Structure of Water at Charged Interfaces: A Molecular Dynamics Study. Langmuir 2014, 30, 8056-8065. 
34. Yang, D.-S.; Zewail, A. H. Ordered water structure at hydrophobic graphite interfaces observed by 4D, ultrafast electron crystallography. Proc. Natl. Acad. Sci. USA 2009, 106, 41224126.

35. Gordillo, M. C.; Marti, J. Molecular dynamics description of a layer of water molecules on a hydrophobic surface. J. Chem. Phys. 2002, 117, 3425-3430.

36. Benderskii, A. V.; Henzie, J.; Basu, S.; Shang, X.; Eisenthal, K. B. Femtosecond Aqueous Solvation at a Positively Charged Surfactant/Water Interface. J. Phys. Chem. B 2004, 108, 14017-14024.

37. Gragson, D. E.; McCarty, B. M.; Richmond, G. L. Surfactant/Water Interactions at the Air/Water Interface Probed by Vibrational Sum Frequency Generation. J. Phys. Chem. 1996, $100,14272-14275$.

38. Xia, X.; Berkowitz, M. L. Electric-Field Induced Restructuring of Water at a PlatinumWater Interface: A Molecular Dynamics Computer Simulation. Phys. Rev. Lett. 1995, 74, 3193. 39. Das, D.; Senapati, S.; Chandra, A. Structure of dipolar liquids near charged solid surfaces: A nonlinear theory based on a density functional approach and Monte Carlo simulations. J. Chem. Phys. 1999, 110, 8129-8138.

40. Ho, T. A.; Striolo, A. Molecular dynamics simulation of the graphene-water interface: comparing water models. Molecular Simulation 2014, 1-11.

41. Gragson, D. E.; McCarty, B. M.; Richmond, G. L. Ordering of Interfacial Water Molecules at the Charged Air/Water Interface Observed by Vibrational Sum Frequency Generation. J. Am. Chem. Soc. 1997, 119, 6144-6152.

42. Kumar, V.; Errington, J. R. Wetting Behavior of Water near Nonpolar Surfaces. J. Phys. Chem. C 2013, 117, 23017-23026.

43. Taherian, F.; Leroy, F.; van der Vegt, N. F. A. Interfacial Entropy of Water on Rigid Hydrophobic Surfaces. Langmuir 2013, 29, 9807-9813.

44. Köfinger, J.; Hummer, G.; Dellago, C. Macroscopically ordered water in nanopores. Proc. Natl. Acad. Sci. USA 2008, 105, 13218-13222.

45. Motakabbir, K. A.; Berkowitz, M. Isothermal compressibility of SPC/E water. J. Phys. Chem. 1990, 94, 8359-8362.

46. Patel, A. J.; Varilly, P.; Chandler, D. Fluctuations of Water near Extended Hydrophobic and Hydrophilic Surfaces. J. Phys. Chem. B 2010, 114, 1632-1637.

47. Schneck, E.; Sedlmeier, F.; Netz, R. R. Hydration repulsion between biomembranes results from an interplay of dehydration and depolarization. Proc. Natl. Acad. Sci. USA 2012, 109, 14405-14409.

48. Tummala, N. R.; Striolo, A. Hydrogen-Bond Dynamics for Water Confined in Carbon Tetrachloride-Acetone Mixtures. J. Phys. Chem. B 2008, 112, 10675-10683.

49. Rosenfeld, D. E.; Schmuttenmaer, C. A. Dynamics of the Water Hydrogen Bond Network at Ionic, Nonionic, and Hydrophobic Interfaces in Nanopores and Reverse Micelles. $J$. Phys. Chem. B 2010, 115, 1021-1031.

50. Boisson, J.; Stirnemann, G.; Laage, D.; Hynes, J. T. Water reorientation dynamics in the first hydration shells of F- and I. Phys. Chem. Chem. Phys. 2011, 13, 19895-19901.

51. Tatsuhiko, O.; Ankur, M.; Seiji, Y.; Hisao, N.; Mischa, B.; Yuki, N. Influence of surface polarity on water dynamics at the water/rutile TiO 2 (110) interface. J. Phys.: Condens. Matter 2014, 26, 244102.

52. Rideg, N. A.; Darvas, M.; Varga, I.; Jedlovszky, P. Lateral Dynamics of Surfactants at the Free Water Surface: A Computer Simulation Study. Langmuir 2012, 28, 14944-14953. 
53. Stirnemann, G.; Castrillon, S. R.-V.; Hynes, J. T.; Rossky, P. J.; Debenedetti, P. G.; Laage, D. Non-monotonic dependence of water reorientation dynamics on surface hydrophilicity: competing effects of the hydration structure and hydrogen-bond strength. Phys. Chem. Chem. Phys. 2011, 13, 19911-19917.

54. Patel, A.; Varilly, P.; Chandler, D.; Garde, S. Quantifying Density Fluctuations in Volumes of All Shapes and Sizes Using Indirect Umbrella Sampling. J Stat Phys 2011, 145, 265275.

55. Lazaridis, T.; Karplus, M. Orientational Correlations and Entropy in Liquid Water. J. Chem. Phys. 1996, 105, 4294-4316.

56. Hernando, J. A.; Blum, L. Density Fluctuations and Entropy. Physical Review E 2000, $62,6577-6583$.

57. Huggins, D. J. Estimating Translational and Orientational Entropies Using the k-Nearest Neighbors Algorithm. J. Chem. Thoery Comput. 2014, 10, 3617-3625.

58. Silverstein, K. A. T.; Dill, K. A.; Haymet, A. D. J. Hydrophobicity in a Simple Model of Water: Entropy Penalty as a Sum of Competing Terms via Full, Angular Expansion. J. Chem. Phys. 2001, 114, 6303-6314.

59. Rosen, M. J., Surfactants and Interfacial Phenomena. 4th ed. ed.; Hoboken, N.J.: Wiley, 2012.

60. Brandriss, S.; Margel, S. Synthesis and Characterization of Self-Assembled Hydrophobic Monolayer Coatings on Silica Colloids. Langmuir 1993, 9, 1232-1240. 
$\underline{\text { Table of Contents Graphic }}$

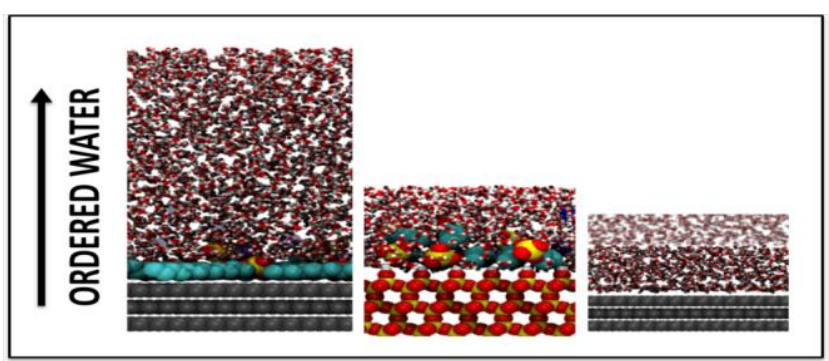

